\title{
Biharmonic Equation on Annulus in a Unit Sphere with Polynomial Boundary Condition
}

\author{
Ikhsan Maulidi ${ }^{1}$, Agah D. Garnadi $^{2}$ \\ ${ }^{1}$ Sumatera Institute of Technology, ikhsan.maulidi@itera.ac.id \\ ${ }^{2}$ Bogor Agricultural University, Agah.garnadi@gmail.com
}

\begin{abstract}
We study the Biharmonic boundary value problem on the annulus with polynomial boundary condition. We utilize an exact algorithms for solving Laplace equation with polynomial Dirichlet conditions with algorithm. The algorithm requires differentiation of the boundary function, but no integration.

Key words and Phrases: Annulus, Biharmonic equation, Dirichlet condition, Laplace equation, polynomial data.
\end{abstract}

\section{INTRODUCTION}

We have known that the harmonic function is a function that satisfies the Laplace equation. The Laplace equation is very important equation and has many applications in the real phenomena such as conductivity equation and wave equation. Biharmonic function is a function which satisfies Bi-Laplace problem. We are interested in studying the Biharmonic equation in this paper.

We study the Biharmonic equation on Annulus with polynomial data in $\mathbb{R}^{3}$. The reason why we use the polynomial data is because it can be writen as the linear combination of the harmonic functions (Axler and Ramey, 1995). We approached the solution by using Lemma that has been formulated by Axler and Ramey (1995) and Lemma by Herzog (2000). In the previous paper, we have studied about Biharmonic in a unit ball in $\mathbb{R}^{3}$ with polynomial data, Maulidi and Garnadi (2016).

Let $x=\left(x_{1}, x_{2}, x_{3}\right) \in \mathbb{R}^{3}$, thus for $\alpha=\left(\alpha_{1}, \alpha_{2}, \alpha_{3}\right)$ of non-negative integers we say $x^{\alpha}$ as monomial $x_{1}^{\alpha_{1}} x_{2}^{\alpha_{2}} x_{3}^{\alpha_{3}}$. The degree of $x^{\alpha}$ is $\alpha_{1}+\alpha_{2}+\alpha_{3}$. A polynomial is said to be homogeneous of degree $m$ if it is a finite linear combination of monomial $x^{\alpha}$ of degree $m ; m=0,1,2, \ldots$. Let $P_{m}$ denotes the vector space of polynomials in $\mathbb{R}^{3}$, homogeneous of degree $m$, and $H_{m}$ is the subspace of harmonic polynomials of degree $m$, where it is satisfies the Laplace equation, then we have property by Axler and Ramey as follows

$$
P_{m}=H_{m}+|x|^{2} P_{m-2},
$$

where $|x|=\sqrt{x_{1}^{2}+x_{2}^{2}+x_{3}^{2}}$.

Let $\Omega$ is a unit sphere in $\mathbb{R}^{3}$, with the boundary

$$
\Gamma=\partial \Omega=\left\{x \in \mathbb{R}^{3} \mid \quad x_{1}^{2}+x_{2}^{2}+x_{3}^{2}=1\right\} .
$$

Let also

then $D^{o}=\Omega-D$ is an annulus region in $\mathbb{R}^{3}$.

$$
D=\left\{x \in \mathbb{R}^{3} \quad ; 0 \leq|x| \leq R<1\right\},
$$

The Biharmonic on Annulus problem can be formulated as follows:

$$
\Delta^{2} u=0 \text { in } D^{o},
$$


2 Maulidi and Garnadi, JMI Vol 13 No 1 Apr 2017, pp. 48-51,doi:10.24198/jmi.v13.n1.11505.48-51

with boundary condition

and

$$
\left.u\right|_{\Gamma}=p_{1} ;\left.\quad u\right|_{D}=p_{2}
$$

where, $p_{1}, p_{2}, q_{1}$ and $q_{2}$ are polynomials.

$$
\left.\Delta u\right|_{\Gamma}=q_{1} ;\left.\quad \Delta u\right|_{D}=q_{2},
$$

\section{TECHNICAL LEMMAS}

These following Lemmas are used to obtain the solution of problem (1)

Lemma 2.1. If $p \in P_{m}$, then the solution to the Dirichlet problem in a unit sphere, $u \in P_{m}$, with boundary data $\left.u\right|_{\Gamma}=p$ is

$$
p_{m}+p_{m-2}+\ldots+p_{m-2 k}
$$

where $k=\left[\frac{m}{2}\right]$ and $p_{m}, p_{m-2}, \ldots, p_{m-2 k}$ are the harmonic polynomials.

The proof of Lemma 2.1 can be seen in Axler and Ramey (1995) and we recommend you to study the algorithm to obtain $p_{j}$ which is a harmonic function.

Lemma 2.2. Given $p \in P_{m}$ and $q \in P_{m+2}$ then there exists $u \in P_{m+2}$ such that

$$
\Delta u(x)=q(x) \quad x \in \Omega \quad \text { and }\left.\quad u\right|_{\Gamma}=p(x) .
$$

Proof. see Herzog (2000).

Lemma 2.3. Let $0<r<s<\infty$, the harmonic function on the annular region in a unit sphere $\{r \leq|x| \leq s\}$ that equals $p \in P_{m}$ on $\{|x|=r\}$ and equals $q \in P_{n}$ on $\{|x|=s\}$ is

$$
\sum_{j=0}^{m} \frac{|x|^{-1-2 j}-s^{-1-2 j}}{r^{-1-2 j}-s^{-1-2 j}} r^{m-j} p_{j}+\sum_{j=0}^{n} \frac{|x|^{-1-2 j}-r^{-1-2 j}}{s^{-1-2 j}-r^{-1-2 j}} s^{n-j} q_{j} .
$$

Proof. The idea for this solution comes from Chapter 10 of [1].

\section{THE MAIN THEOREM}

Teorema 3.1. Given $p_{1} \in P_{m_{1}}, p_{2} \in P_{m_{2}}, q_{1} \in P_{n_{1}}$, and $q_{2} \in P_{n_{2}}$ then there exists $u_{1} \in P_{m}$ and $u_{2} \in P_{n}, m=\max \left(m_{1}, m_{2}\right)$ and $n=\max \left(n_{1}, n_{2}\right)$, such that $u=u_{1}+u_{2}$ is the solution of (1).

Proof. Here we give the algorithm to solve the problem (1). The Biharmonic type problem was presented in (1) can be written in two subproblem:

$$
\Delta u=v \quad \text { in } \quad D_{o} ;
$$

with conditions

$$
\begin{aligned}
& \left.u\right|_{\Gamma}=p_{1}, \\
& \left.u\right|_{D}=p_{2} .
\end{aligned}
$$

And

$$
\Delta v=0 \quad \text { in } \quad D_{o}
$$


with conditions

$$
\begin{aligned}
& \left.v\right|_{\Gamma}=q_{1}, \\
& \left.v\right|_{D}=q_{2} .
\end{aligned}
$$

The solution of problem (3) can be formulated if we had solved the problem (4). The solution of problem (4), $v \in P_{n}, n=\max \left(n_{1}, n_{2}\right)$ can be obtained by using Lemma 2.3 which is a harmonic polynomials function. By setting $r=R$ and $s=1$, then we have

$$
v=\sum_{j=0}^{n_{1}} \frac{|x|^{-1-2 j}-1}{R^{-1-2 j}-1} R^{n_{1}-j} q_{1 j}+\sum_{j=0}^{n_{2}} \frac{|x|^{-1-2 j}-R^{-1-2 j}}{1-R^{-1-2 j}} q_{2 j},
$$

where $q_{1 j}$ and $q_{2 j}$ are the harmonic polyomials which have degree $j . q_{1 j}, q_{2 j} \in H_{j}$.

By using the principle of linear superposition, suppose that the solution of (3) is $u=$ $u_{1}+u_{2}$, where $u_{1}$ is the solution of this problem:

$$
\Delta u_{1}=0 \quad ;\left.u_{1}\right|_{\Gamma}=p_{1} \text { and }\left.u_{1}\right|_{D}=p_{2},
$$

and $u_{2}$ is the solution of this problem:

$$
\Delta u_{2}=v \quad ;\left.u_{2}\right|_{\Gamma}=0 \text { and }\left.u_{2}\right|_{D}=0 .
$$

The solution of (5) can be obtained by using Lemma 2.3 which is the harmonic polynomials function. Where $u_{1} \in P_{m}, m=\max \left(m_{1}, m_{2}\right)$. Next, by solving problem (6) when the solution of this problem is guaranteed by Lemma 2.2, so we have $u_{2} \in P_{n}$. Therefore this completes the proof of Theorem 3.1.

\section{CONCLUSION}

The Biharmonic on annulus in a unit sphere with polynomial boundary condition that presented in (1) has a solution. The solution can be obtained by using the algorithm and the existence of this solution is guaranteed by using Lemma 2.2 and Lemma 2.3.

\section{REFERENCES}

[1] Axler, S. Bourdon P. and Ramey, W. K., Harmonic Function Theory, Graduate Text in Mathematics, Springer-Verlag, 1992.

[2] Axler, S. and Ramey, W, "Harmonic polynomials and Dirichlet type problems", Proc. Amer. Math. Soc. 123(12) (1995), 3765 - 3773

[3] Herzog,G., "Polynomials solving Dirichlet boundary value problems", Proc. Amer. Math. Soc. 107(10) (2000), 934-936.

[4] Maulidi, I. and Garnadi, A.D, "Biharmonic equation in a Unit Ball in $\mathbb{R}^{3}$ with Polynomial Data", Proc. Basic Malang. (2016). 